Original Research Paper

\title{
Control of Bubble Formation at the Optical Fiber Tip by Analyzing Ultrasound Acoustic Waves
}

\author{
${ }^{1}$ Viacheslav Viacheslavovich Kazakov, ${ }^{2}$ Vladimir Isaakovich Yusupov, \\ ${ }^{2}$ Viktor Nikolaevich Bagratashvili, ${ }^{1}$ Anton Ivanovich Pavlikov and ${ }^{1}$ Vladislav Antonievich Kamensky \\ ${ }^{1}$ Institute of Applied Physics of the Russian Academy of Sciences, 46 Ulyanov Street, 603950, Nizhny Novgorod, Russia \\ ${ }^{2}$ Institute of Photon Technologies of Federal Scientific Research Centre "Crystallography and Photonics" of the Russian \\ Academy of Sciences, 2 Pionerskaya Street, 142190, Troitsk, Moscow, Russia
}

Article history

Received: 24-10-2016

Revised: 25-10-2016

Accepted: 01-11-2016

Corresponding Author: Viacheslav Viacheslavovich Kazakov

Institute of Applied Physics of the Russian Academy of Sciences, 46 Ulyanov Street, 603950, Nizhny Novgorod,

Russia

E-mail:kazak@appl.sci-nnov.ru

\begin{abstract}
The problems of controlling the processes of generation of acoustic waves and microbubbles at the optical fiber tip of moderatepower surgical lasers with wavelengths of $1.9 \mu \mathrm{m}$ and $0.97 \mu \mathrm{m}$ using active and passive acoustic methods are considered. It is shown that microbubbles of various sizes can be detected with an ultrasound method, in which the interaction area is recorded simultaneously at two frequencies. By measuring noise with a receiving transducer of an ultrasonic locator, we determine the frequency range of the acoustic signals occurring when large diameter bubbles, including gas-vapor bubbles, collapse. Model experiments are conducted to detect noise and bubbles in water and in a water-saturated $1.5 \%$ agarose phantom at various laser powers with an optical fiber tip having a strongly absorbing coating.
\end{abstract}

Keywords: Laser Heating, Dual-Frequency Ultrasound, Acoustic Noise, Bubble Detection, Optical Fiber with Strongly Absorbing Coating

\section{Introduction}

Local high-temperature heating of biological tissue by optical radiation is widely used in laser surgery, allowing to increase the speed of tissue cutting, decrease the width of the cutting area and to reduce wound healing time (Romanos, 2013; Kuznetsova et al., 2015; Venugopalan et al., 1996). In dentistry, this method is employed for enamel removal, in vascular surgery - for vessel ablation (Altshuler et al., 2001; Romanos et al., 2015; Theivacumar et al., 2008). New treatment techniques based on the formation of laser channels in biological tissue by a heated fiber tip are used in neurosurgery (Sandler et al., 2002). The interaction of optical radiation with biological tissue is multifaceted and, in particular, is accompanied by the occurrence of acoustic noise and the formation of bubbles (Mettin, 2007; Yusupov et al., 2014). Cavitation bubbles can effectively disinfect biological tissues, as has been reported in several studies (Lifshitz et al., 2005; Verhaagen and Rivas, 2016), so they attract special attention.

Naturally, the control of the processes occurring in the area of laser-induced tissue destruction becomes an important task. The most popular method of studying the appearance and formation of bubbles at the optical fiber tip is the high-speed video monitoring in water or in a thin layer of a biological tissue phantom illuminated by an external source (Asshauer et al., 1997; Lü and Li, 2011; Yusupov et al., 2010; Skrypnik, 2015; Belikov et al., 2015). Using this method, specificities of visible-size bubble generation at the fiber tip with a strongly absorbing coating were elucidated and the formation of channels, acoustic flows, was detected. Of considerable practical interest are methods of control that allow to more easily evaluate the cutting process, for example, by acoustic noise and to determine the formation, presence and parameters of moving microbubbles, whose visualization is difficult or impossible. The generated acoustic noise results from the occurrence, interaction and collapse of bubbles of various sizes in the bulk of the medium or on its surface and the processes are almost simultaneous, making it difficult to categorize the bubbles by size and define their origin. Part of this problem can be solved with ultrasound techniques that are based on remote excitation of bubbles at resonant frequencies and detection of waves generated by the 
bubbles, the level of amplitude of the waves being indicative of the bubbles (Kazakov et al., 2016; Chen et al., 2006; Maksimov, 1997; Leighton et al., 1996; Vos, 2010). The use of the nonlinear effect, the parametric excitation of bubbles at the subharmonic frequency, significantly improves the reliability of bubble detection (Mansfel'd et al., 2005; 2010; Daeichin et al., 2015; Zhang and Li, 2015; Zhang et al., 2015; Wu et al., 2004; Lo et al., 2004; Phelps and Leighton, 1996).

In this study, we investigate the possibility of controlling the processes of formation of microbubbles of different sizes under different conditions by investigation the area of irradiation with ultrasound waves at two frequencies in the megahertz range and by analyzing the generated noise level.

\section{Materials and Methods}

\section{Experimental Setup}

A schematic representation of the experimental setup is shown in Fig. 1. The optical radiation sources were a Alta-ST Laser System from Dental Photonics (wavelength $\lambda=0.97 \mu \mathrm{m}$, maximum power $\mathrm{P}=22 \mathrm{~W}$, absorption coefficient in water is $0.47 \mathrm{~cm}^{-1}$ ) and a LS1.9 laser from IRE-Polus $\left(1.9 \mu \mathrm{m}, 3 \mathrm{~W}, 92 \mathrm{~cm}^{-1}\right)$ with a silica optical fiber $\mathrm{S} 1$ with a diameter of $400 \mu \mathrm{m}$ (Deng et al., 2012). Prior to experiments at $\lambda=0.97 \mu \mathrm{m}$, a highly absorbing coating (Dental Photonics compound) was applied onto the tip of the optical fiber, resulting in heating of the fiber tip by laser radiation. Specially developed in IAP RAS ultrasound locator was used for bubble detection, in which a transducer $\mathrm{T} 1$ was excited in a long-pulse regime simultaneously at two frequencies $f_{1}=2.92 \mathrm{MHz}$ and $f_{2}=0.3 \mathrm{MHz}$ (resonant frequencies of the longitudinal and radial oscillation modes of the piezoelectric element of transducer T1). A simplified calculation of an approximate bubble radius as a function of resonant frequency has been presented in (Mansfel'd et al., 2005; Plesset and Prosperetti, 1977; Ainslie and Leighton, 2011). For sub-harmonic frequencies $f_{1} / 2$ and $f_{2} / 2$ the bubbles have radii of $2.6 \mu \mathrm{m}$ and $22 \mu \mathrm{m}$. The ultrasonic repetition pulse frequency was $5 \mathrm{~Hz}$. The emitting $\mathrm{T} 1$ and receiving $\mathrm{T} 2$ ultrasound transducers were $7 \mathrm{~mm}$ in diameter and $20 \mathrm{~mm}$ long. Their emitting and receiving characteristics were measured by an impedance meter LCR-78110G (GW Instek). Signals received from the intersection area of ultrasound beams of the transducers $\mathrm{T} 1$ and $\mathrm{T} 2$ were registered by an AKIP-75244B USB-oscilloscope, which used synchronization from the ultrasound locator and a sampling frequency of 31.25 MHz. Fifty measurement cycles of $32.8 \mu \mathrm{s}$ duration each (1024 counts) were performed. The spectrum analysis was carried out with a resolution of $0.03 \mathrm{MHz}$ in the frequency range $0 \div 5 \mathrm{MHz}$. The analysis region was set by selecting distances $d_{1}=15 \mathrm{~mm}$ and $d_{2}=4 \div 10 \mathrm{~mm}$ (Fig. 1). The measurements were made either in a water-filled container S3 with sizes $300 \times 220 \times 200 \mathrm{~mm}$ (Fig. 1a) or in a biological tissue phantom placed in a cylinder $70 \mathrm{~mm}$ in diameter and $30 \mathrm{~mm}$ in height, containing $98.5 \%$ water and $1.5 \%$ agarose (Fig. $1 \mathrm{~b}$ ).

The T1 and T2 transducers were attached to the lateral sides of the sample in the center and the optical fiber was inserted from above.
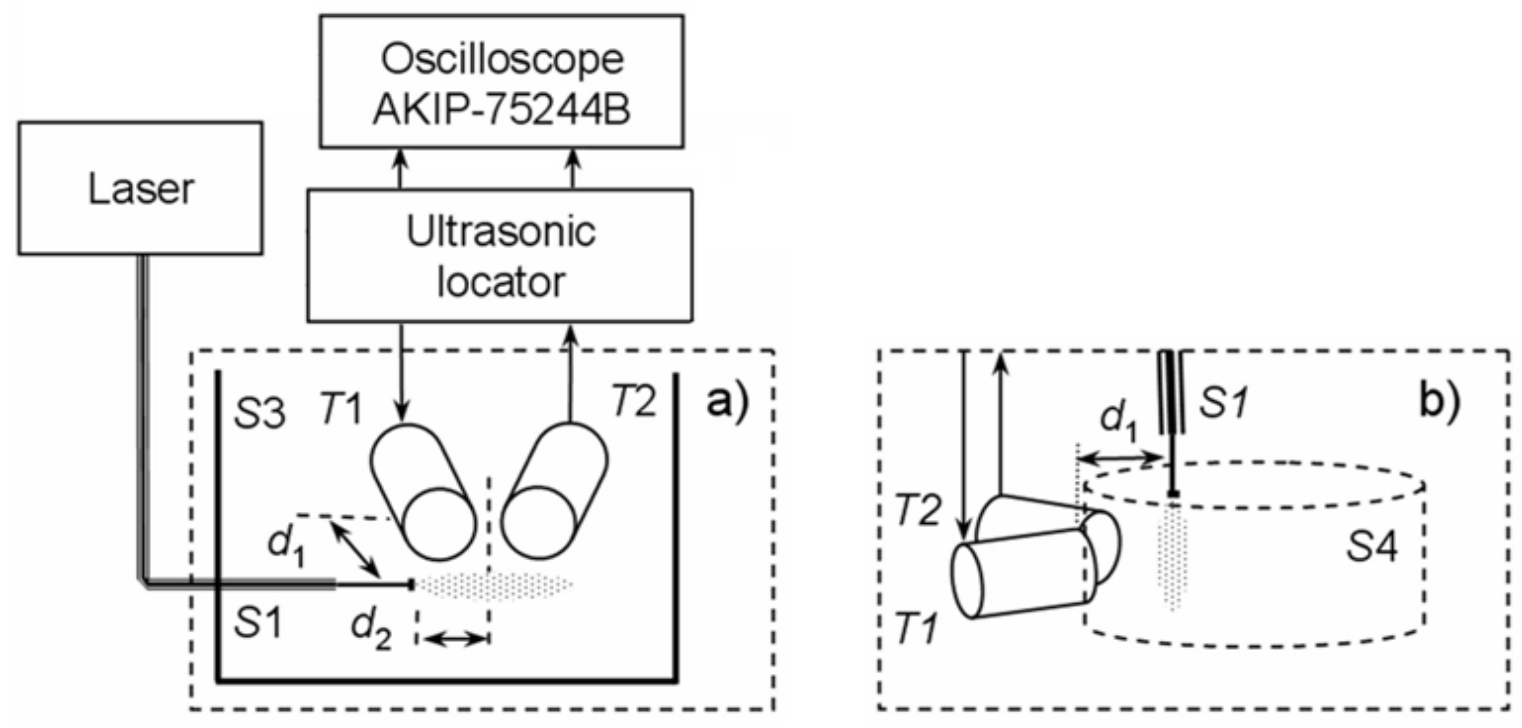

Fig. 1. (a) Diagram of the experimental setup (b) diagram of the experimental setup 


\section{Results and Discussion}

Figure 2 shows an oscillogram of the signal (one cycle) from the output of transducer $\mathrm{T} 2$, demonstrating a multi-frequency signal.

In addition to the signals from generated ultrasound waves, the $\mathrm{T} 2$ transducer receives acoustic noise originating from the formation and collapse of bubbles inside the medium or on its surface. When a fiber tip with a highly absorbing coating is used, bubbles with sizes up to a few tens of microns form and collapse on the surface of the coating due to explosive boiling. Part of these bubbles detach from the tip and collapse some distance from it. Also, slowly growing vapor-gas bubbles may form on the tip. They may grow to sizes comparable with the tip diameter, detach from the tip and rise to the surface to collapse. When an uncoated fiber tip is used, at laser radiation with $\lambda=1.9 \mu \mathrm{m}$ bubbles with sizes reaching up to several $\mathrm{mm}$ are formed and collapse in the medium due to thermal cavitation. As a result of these processes, the broadband noise is generated in the medium, which is comparable to or even exceeds the level of signals detectable by ultrasonic diagnostic. The upper frequency values relevant to the analysis can reach $0.4 \div 0.5 \mathrm{MHz}$ and in this frequency range the noise can mask signals for frequencies $f_{2}$ and $f_{2} / 2$, making it difficult to detect microbubbles with sizes of tens of microns. Obviously, the higher the detection frequency, the lower is the effect of acoustic noise on the accuracy of microbubbles detection.

A specific feature of the analysis of signals received during ultrasonic detection is that the duration of each cycle being recorded ( $32.8 \mu \mathrm{s})$ is significantly less than the duration of pulsed signals of acoustic noise and the recording time is not synchronized with their appearance. As a result, the contribution of acoustic noise to the change of the level of harmonics and subharmonics of the received ultrasound waves is significantly reduced, depends on the frequency range of the analysis and is revealed only in the rare moments of the collapse of vapor-gas bubbles, because their spectrum at high frequencies reaches a few MHz. Since the receiving transducer $\mathrm{T} 2$ is a broadband one, it can be used to analyze changes in the amplitude of spectral components not only at the frequencies used for ultrasound detection, but also at an "intermediate" frequency $f_{3}$, providing additional information on the current level of acoustic noise.

Figure 3 shows the dependences of the amplitude change of the received signals at frequencies $f_{1}=$ $2.92 \mathrm{MHz}$ and $f_{2}=0.30 \mathrm{MHz}$, their sub-harmonics $f_{1} / 2=$ $1.46 \mathrm{MHz}$ and $f_{2} / 2=0.15 \mathrm{MHz}$ and at the "intermediate" frequency $f_{3}=0.22 \mathrm{MHz}$ selected to measure acoustic noise, for measurements in water (Fig. 3a) and in an agarose phantom (Fig. 3b). Lasers with $\lambda=0.97 \mu \mathrm{m}$ (coated tip, $\mathrm{P}=22 \mathrm{~W}$ ) and $\lambda=1.9 \mu \mathrm{m}$ (non-coated tip, $\mathrm{P}=$ $3 \mathrm{~W})$ were used in the experiments. Fifty cycles were recorded during the measurements. The first 8-15 cycles were recorded with the laser turned off and then the laser was turned on. At $d_{2} \leq 4 \mathrm{~mm}$ (Fig. 1) a reflection from the optical fiber tip is present in the received signal. To eliminate its effect on the measurement result, the calculation method described in ( $\mathrm{Lü}$ and $\mathrm{Li}, 2011)$ was used, in which the first cycle was subtracted from all subsequent cycles.

It can be clearly seen from Fig. $3 a$ and $3 b$ that the results of measurements in water and in the biological tissue phantom qualitatively coincide. The sharp increase in the amplitude of the subharmonic frequency $f_{1}$ indicates that the formation of micron-sized bubbles begins immediately after the laser is turned on. There are moments when the number of bubbles drops considerably. By changes in the amplitude of the subharmonic frequency $f_{1}$ it is possible to detect the periodicity of the micron bubbles formation. Bubbles with sizes of about twenty microns in gel behave more stable, their number is approximately constant over time. The values of the amplitudes for frequencies $f_{1}$ and $f_{2}$ reflect both the changes in impedance of the medium at the detection site due to bubble formation and the changes in the structure (in the agarose phantom). That is why the dependence of the amplitude changes over time is more complex and it is difficult to reliably determine the level of bubble generation by changes in the signal amplitudes at frequencies $f_{1}$ and $f_{2}$. The change in the harmonic amplitude at frequency $f_{3}$ reflects well the occurrence of noise when the laser is turned on and the noise is quite stable. It is obvious that only the analysis of signal changes at all of these frequencies can provide reliable information on the results of interaction of optical radiation with the medium. Changes in the "structure" in the interaction region can be determined by ultrasound at frequencies of megahertz range, the formation of bubbles with sizes from several to tens of microns can be detected by measuring the level of sub-harmonics and the progress of the interaction of optical radiation with a fluid or biological tissue can be monitored by measuring the noise level at a frequency chosen in the range of $0.1 \div 0.5 \mathrm{MHz}$.

Figure 4 shows the dependences of the signal amplitudes for various frequencies versus power and wavelength of laser radiation in water and in an agarose phantom. Averaging was performed for a period of $3 \mathrm{~s}$. For a laser with $\lambda=0.97 \mu \mathrm{m}$ (thin lines in Fig. 4) the changes in the amplitude at all frequencies clearly indicate that at $\mathrm{p}>10 \mathrm{~W}$ the coating of the fiber tip begins to degrade. Additional measurements showed that the time at which the absorbing coating remains 
stable at high powers is limited to tens of seconds. Increasing the power from 1 to $3 \mathrm{~W}$ for a laser with high attenuation $(\lambda=1.9 \mu \mathrm{m}$, thick lines in Fig. 4a) leads to an almost linear increase in signal amplitudes at frequencies $f_{1} / 2$ and $f_{2} / 2$ and hence to a number of bubbles in the $1.5 \div 20 \mu \mathrm{m}$ range. This "linear" character of the dependence of the sub-harmonic amplitude level on power is important for practical use, as it permits to regulate saturation of water with microbubbles of a particular size by varying the power of laser radiation.

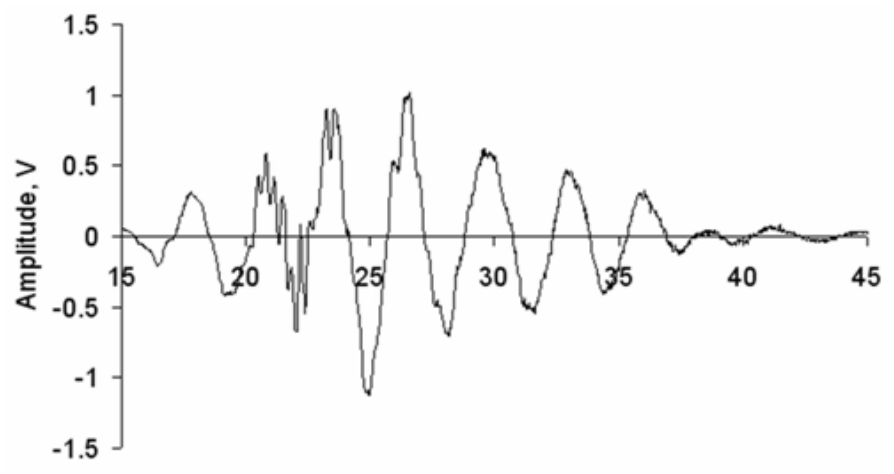

Time, us

Fig. 2. Typical oscillogram of the signal for dual-frequency ultrasound detection
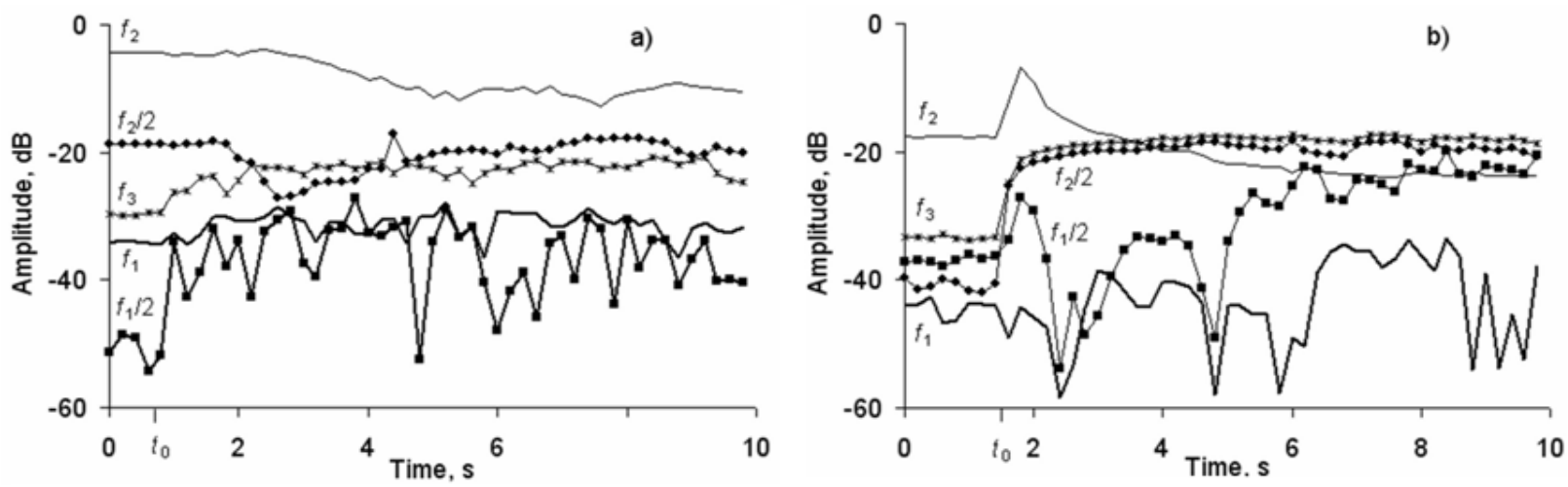

Fig. 3. Dependences of the change of amplitudes of the received acoustic signals at various frequencies for measurements in water (a) and in an agarose phantom (b)
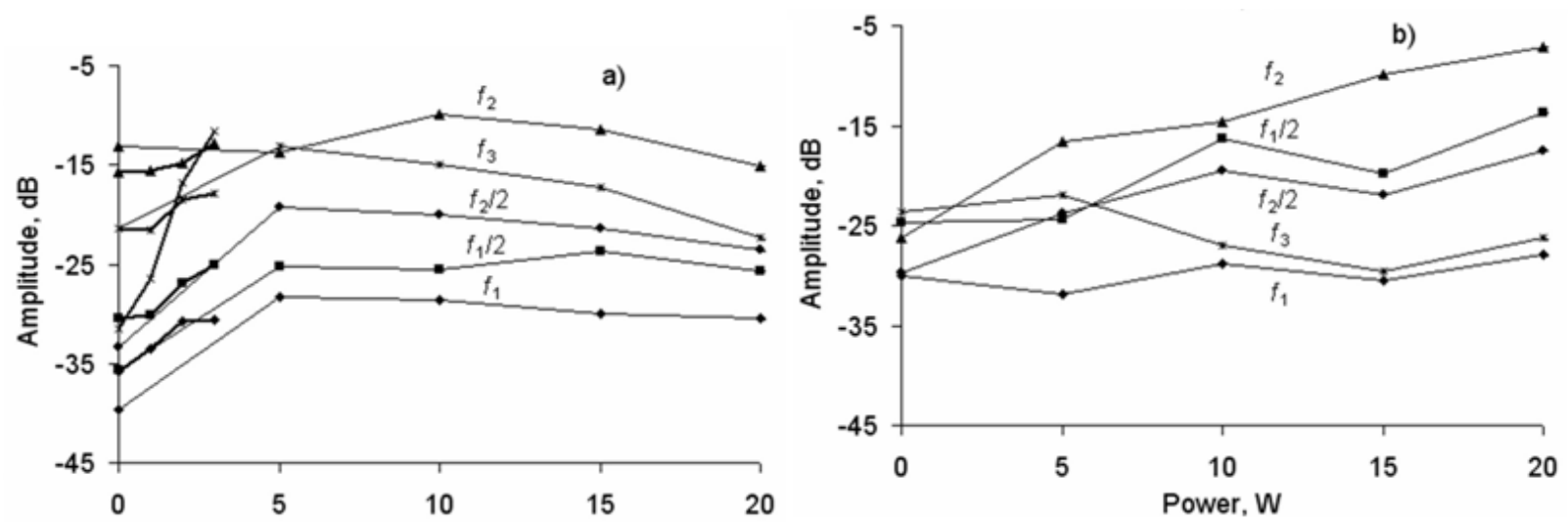

Fig. 4. The dependence of amplitudes of received acoustic signals at various frequencies on power and wavelength of laser radiation in water (a) and in an agarose phantom (b) 

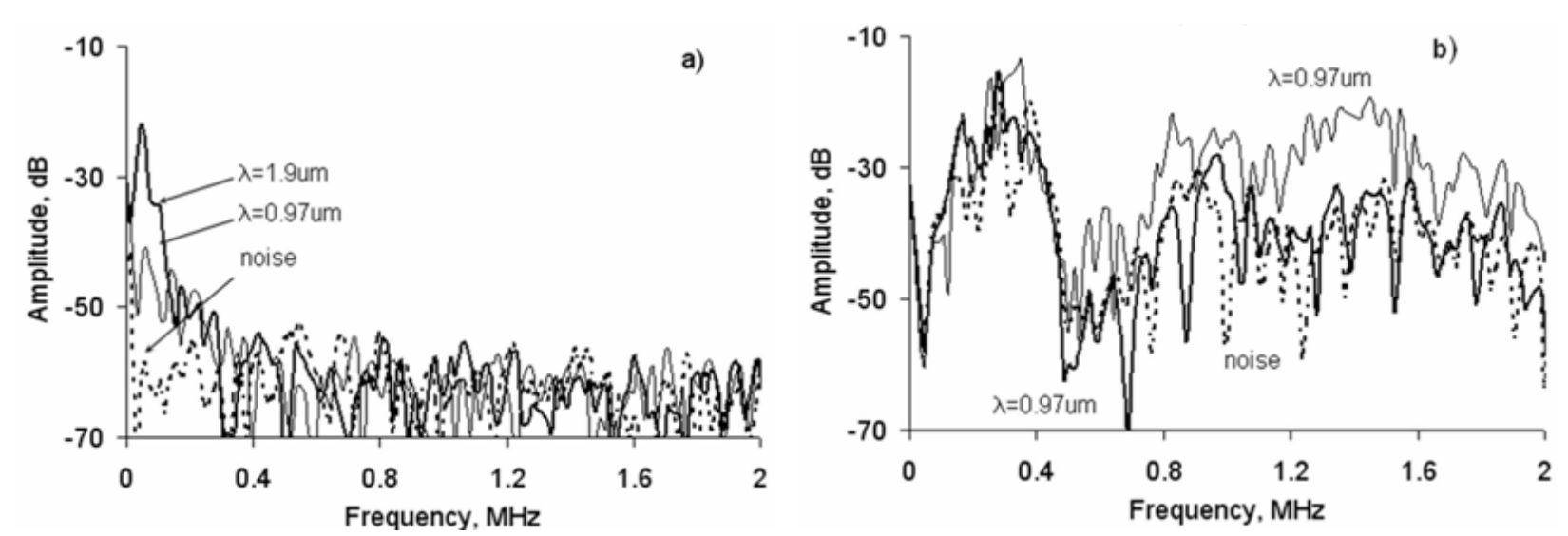

Fig. 5. Signal spectra at laser irradiation in water (a) and in an agarose phantom (b) for various $\mathrm{P}$ and $\lambda$

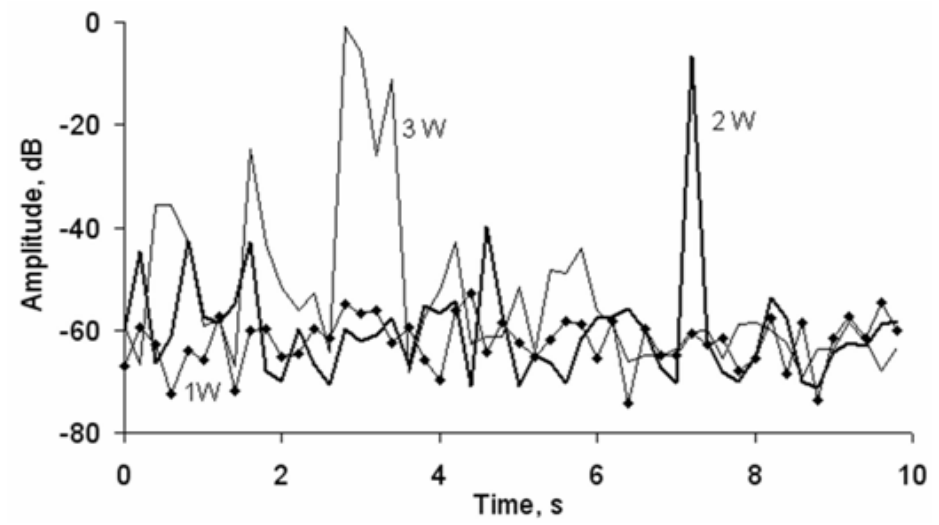

Fig. 6. Changes in the signal amplitude at the frequency of $0.22 \mathrm{MHz}$ when irradiating in water for various powers of laser radiation at $\lambda=1.9 \mu \mathrm{m}$

The maximum values of signal spectra for measurements in water and in an agarose phantom are shown in Fig. 5a and $5 \mathrm{~b}$ for $\lambda=0.97 \mu \mathrm{m}(\mathrm{P}=20 \mathrm{~W})$ and $\lambda=1.97 \mu \mathrm{m}(\mathrm{P}=3 \mathrm{~W})$. It is evident from these figures that when irradiating in water the maximum energy of the received signals is in the frequency range of $0 \div 0.4 \mathrm{MHz}$ and the peak amplitudes may exceed the noise up to $30 \mathrm{~dB}$. The collapse of gas-vapor bubbles leads to a short-term increase in noise up to $40 \mathrm{~dB}$ and the upper frequency value can increase up to a few megahertz. This effect is manifest in a sharp (during one ultrasound signal, i.e., $0.2 \mathrm{~s}$ ) increase in amplitudes of individual spectral components. As an example, on Fig. 6 shown the amplitude change of the received signal at the $f_{3}$ frequency for measurements in water $(\lambda=1.9 \mu \mathrm{m})$. It can be seen that the level of the received signal increases with increasing laser power and the signal bursts indicating the formation and collapse of vapor-gas bubbles are more frequent.

The spectrum of the signal received by the transducer $\mathrm{T} 2$ in the agarose phantom is significantly different from that measured in water (Fig. 5b). This is due to the fact that in the agarose phantom, in addition to the reflection from the fiber tip and bubbles, there is also a reflection from the altered structure of the phantom, since the optical radiation quite quickly forms a cavity filled with water and gas in the phantom near the fiber tip. Upon reaching a certain size, the reflection of ultrasound waves from this cavity becomes dominant and the signals from the fiber tip and bubbles are masked.

\section{Conclusion}

It has been experimentally demonstrated that by using a dual-frequency ultrasound locator and by additionally passively receiving acoustic signals with the receiver of the same locator, it is possible to monitor processes associated with laser-induced generation of bubbles near the optical fiber tip. This method allows the detection of: (1) The formation of bubbles of different sizes, ranging from a few to tens of microns, (2) the moment when the laser is turned on, (3) the collapse of vapor-gas bubbles and (4) the degradation of the fiber tip coating. It has been shown that only the multi-frequency signal analysis is able to provide reliable information on 
the current process of interaction of optical radiation with the medium. Furthermore, this process can be controlled. For example, by varying the laser power according to the level of subharmonic amplitude, one can automatically control the saturation of the medium (water or biological tissue) with bubbles of a particular size. It has also been shown that the laser radiation at $\lambda=1.9 \mu \mathrm{m}$, having a significantly lower (7-10 times) radiation power, generates the same level of acoustic noise as the laser radiation at $\lambda=0.97 \mu \mathrm{m}$.

\section{Acknowledgement}

The authors would like to acknowledge financial support from grant 14-15-00840 of the Russian Scientific Fund.

\section{Author's Contributions}

Viacheslav Viacheslavovich Kazakov: Designed of ultrasonic device and transducers, performed of the experiments, performed analysis of data, wrote the article.

Vladimir Isaakovich Yusupov: Participated in experimental plan, performed the experiments, tested of lasers, interpreted the data, contributed to the writing of the article.

Viktor Nikolaevich Bagratashvili: Supervised the research programme and gave important feedback on the research result, critical reviewed of the scientific content of the article.

Anton Ivanovich Pavlikov: Made phantoms containing water and agarose, performed of the experiments, edited of article.

Vladislav Antonievich Kamensky: Planned and coordinated the research, tested of lasers and optical fiber tips, analyzed the results, contributed to the writing of the article.

\section{Ethics}

This article is original and contains unpublished material. The corresponding author confirms that all of the other authors have read and approved the manuscript and no ethical issues involved.

\section{References}

Ainslie, M.A. and T.G. Leighton, 2011. Review of scattering and extinction cross-sections, damping factors and resonance frequencies of a spherical gas bubble. J. Acoust. Soc. Am., 130: 3184-3208. DOI: 10.1121/1.3628321

Altshuler, G.B., A.V. Belikov and Y.A. Sinelnik, 2001. A laser-abrasive method for the cutting of enamel and dentin. Lasers Surg. Med., 28: 435-444. DOI: $10.1002 / \mathrm{lsm} .1071$
Asshauer, T., G. Delacretaz, E.D. Jansen, A.J. Welch and M. Frenz, 1997. Pulsed holmium laser ablation of tissue phantoms: correlation between bubble formation and acoustic transients. Applied Phys. B, 65: 647-657. DOI: 10.1007/s003400050327

Belikov, A.V., A.V. Skrypnik and K.V. Shatilova, 2015. Comparison of diode laser in soft tissue surgery using continuous wave and pulsed modes in vitro. Front. Optoelectron., 8: 212-219.

DOI: $10.1007 / \mathrm{s} 12200-015-0517-8$

Chen, S., R. Kinnick, J.F. Greenleaf and M. Fatemi, 2006. Difference frequency and its harmonic emitted by microbubbles under dual frequency excitation. Ultrasonics, 44: e123-e126. DOI: 10.1016/j.ultras.2006.07.009

Daeichin, V., J.G. Bosch and A. Needles, 2015. Subharmonic, non-linear fundamental and ultraharmonic imaging of microbubble contrast at high frequencies. Ultrasound Med. Biol., 41: 486-497. DOI: 10.1016/j.ultrasmedbio.2014.10.003

Deng, R., Y. He, Y. Qin, Q. Chen and L. Chen, 2012. Measuring pure water absorption coefficient in the near-infrared spectrum (900-2500 nm). J. Remote Sens., 16: 192-206.

Kazakov, V.V., A.G. Sanin and V.A. Kamensky, 2016. On the possible ultrasonic inspection of microbubbles generated by the optical fiber tip. J. Innov. Opt. Health Sci., 9: 1650013-1650020.

DOI: $10.1142 / \mathrm{S} 1793545816500139$

Kuznetsova, D.S., M.M. Karabut, V.V. Elagin, M.A. Shakhova and V.I. Bredikhin et al., 2015. Comparative analysis of biotissue laser resection using strongly absorbing optical fiber tips. Opt. Photon. J., 5: 1-5. DOI: 10.4236/opj.2015.51001

Leighton, T.G., A.D. Phelps, D.G. Ramble and D.A. Sharpe, 1996. Comparison of the abilities of eight acoustic techniques to detect and size a single bubble. Ultrasonics, 34: 661-667.

DOI: 10.1016/0041-624X(96)00053-4

Lifshitz, T., J. Levy, I. Klemperer and S. Levinger, 2005. Anterior chamber gas bubbles after corneal flap creation with a femtosecond laser. J. Cataract. Refract. Surg., 31: 2227-2229.

DOI: $10.1016 /$ j.jcrs.2004.12.069

Lo, M.T., J. Tsao and S. Lin, 2004. Using the correlation property of subharmonic response as an index of cavitation of microbubbles. Proceedings of the IEEE Ultrasonics Symposium, Aug. 23-27, IEEE Xplore Press, pp: 1383-1386. DOI: 10.1109/ULTSYM.2004.1418055

Lü, T. and Z.J. Li, 2011. Underwater holmium-laserpulse-induced complete cavitation bubble movements and acoustic transients. Chinese Sci. Bull., 56: 1226-1229. DOI: 10.1007/s11434-011-4367-5 
Maksimov, A.O., 1997. On the subharmonic emission of gas bubbles under two-frequency excitation. Ultrasonics, 35: 79-86.

DOI: $10.1016 / \mathrm{S} 0041-624 \mathrm{X}(96) 00086-8$

Mansfel'd, A.D., D.A. Mansfel'd and A.M. Reyman, 2005. Abilities of nonlinear acoustic methods in locating gas bubbles in biological tissues. Acoustical Phys., 51: 209-217. DOI: 10.1134/1.1884496

Mansfel'd, A.D., G.P. Volkov, A.G. Sanin and I.A. Vladimirov, 2010. Pulsed ultrasonic spectroscopy of gas bubbles. Acoustical Phys., 56: 290-298. DOI: 10.1134/S106377101003005X

Mettin, R., 2007. From a Single Bubble to Bubble Structures in Acoustic Cavitation. In: Oscillations, Waves and Interactions, Kurz, T., U. Parlitz and U. Kaatze (Eds.), Universitatsverlag Gottingen, pp: 171-198.

Phelps, A.D. and T.G. Leighton, 1996. High-resolution bubble sizing through detection of the subharmonic response with a two-frequency excitation technique. J. Acoust. Soc. Am., 99: 1985-1992. DOI: $10.1121 / 1.415385$

Plesset, M. and A. Prosperetti, 1977. Bubble dynamics and cavitation. Ann. Rev. Fluid Mech., 9: 145-185. DOI: 10.1146/annurev.fl.09.010177.001045

Romanos, G.E., 2013. Diode laser soft-tissue surgery: Advancements aimed at consistent cutting, improved clinical outcomes. Compend. Continu. Educ. Dent., 34: 752-757. PMID: 24571504

Romanos, G.E., A.V. Belikov, A.V. Skrypnik, F.I. Feldchtein and M.Z. Smirnov et al., 2015. Uncovering dental implants using a new ThermoOptically Powered (TOP) technology with tissue aircooling. Lasers Surg. Med., 47: 411-420. DOI: $10.1002 / 1 \mathrm{sm} .22360$

Sandler, B.I., V.M. Chudnovskiy, V.I. Yusupov and Y. Galin, 2002. Treating of compression and reflectory lumbar osteochondritis with the method of polycanal laser decompression. Bull. Physiol. Pathol. Respirat., 11: 46-49.

Skrypnik, A.V., 2015. Fiber optoacoustic converter of laser radiation. Izvestiya Vysshikh Uchebnykh Zavedeniy. Priborostroenie., 58: 385-392.
Theivacumar, N.S., D. Dellagrammaticas, R.J. Beale, A.I.D. Mavor and M.J. Gough, 2008. Factors influencing the effectiveness of Endovenous Laser Ablation (EVLA) in the treatment of great saphenous vein reflux. Europ. J. Vasc. Endovasc. Surg., 35: 119-123.

DOI: $10.1016 /$ j.ejvs.2007.08.010

Venugopalan, V., N.S. Nishioka and B.B. Miki, 1996. Thermodynamic response of soft biological tissues to pulsed infrared-laser irradiation. Biophys. J., 70: 2981-2993. PMID: 8744336

Verhaagen, B. and D.F. Rivas, 2016. Measuring cavitation and its cleaning effect. Ultrasonics Sonochemistry, 29: 619-628.

DOI: $10.1016 /$ j.ultsonch.2015.03.009

Vos, H.J., 2010. Single microbubble imaging. Erasmus Medical Center. Rotterdam, Netherlands.

Wu, C.Y., M.T. Lo, J. Tsa, D.H. Tsai and Y.C. Chang et al., 2004. The short-pulse subharmonic response of microbubbles based on a two-frequency approximation. Proceedings of the IEEE Ultrasonics Symposium, Aug. 23-27, IEEE Xplore Press, pp: 1379-1382.

DOI: 10.1109/ULTSYM.2004.1418053

Yusupov, V.I., V.M. Chudnovskii and V.N. Bagratashvili, 2010. Laser-induced hydrodynamics in watersaturated biotissues. 1. Generation of bubbles in liquid. Laser Phys., 20: 1641-1646. DOI: $10.1134 / \mathrm{S} 1054660 \mathrm{X} 1014001 \mathrm{X}$

Yusupov, V.I., V.V. Bulanov, V.M. Chudnovskii and V.N. Bagratashvili, 2014. Laser-induced hydrodynamics in water-saturated tissue: III. Optoacoustic effects. Laser Phys., 24: 015601-015601. DOI: $10.1088 / 1054-660 X / 24 / 1 / 01560$

Zhang, Y. and S. Li, 2015. Acoustical scattering cross section of gas bubbles under dual-frequency acoustic excitation. Ultrasonics Sonochem., 26: 437-444. DOI: 10.1016/j.ultsonch.2015.02.018

Zhang, Y., X. Du, H. Xian and Y. Wu, 2015. Instability of interfaces of gas bubbles in liquids under acoustic excitation with dual frequency. Ultrasonics Sonochem., 23: 16-20.

DOI: 10.1016/j.ultsonch.2014.07.021 